\title{
Eidgenössische Volksinitiative "Für eine starke Pflege»
}

\author{
Helena Zaugg a und Yvonne Ribi ${ }^{b}$ \\ a Präsidentin des Schweizer Berufsverbands der Pflegefachfrauen und Pflegefachmänner SBK \\ ${ }^{b}$ Geschäftsführerin des Schweizer Berufsverbands der Pflegefachfrauen und Pflegefachmänner SBK
}

Um die Lesbarkeit zu

erleichtern, werden die

männliche und die

weibliche Form

unregelmässig

abwechselnd verwendet

Massiv zu tiefe Ausbildungszahlen, stetig steigender Zeitdruck und schwierige Arbeitsbedingungen verschlechtern die Attraktivität des Pflegeberufes zunehmend. Der Schweizer Berufsverband der Pflegefachfrauen und Pflegefachmänner SBK lancierte im Januar 2017 die Pflegeinitiative als Massnahme zur Sicherung der pflegerischen Versorgungsqualität. Die Bevölkerung zeigt Verständnis für die Forderungen, und die FMH unterstützt die Initiative.

\section{Ausgangslage}

Bereits vor zehn Jahren wurde prognostiziert, dass die Kosteneinsparungsstrategien in den Spitälern längerfristig einen Qualitätsabbau erwarten lassen, der sich

\section{Zusammenfassung}

Hintergrund der Pflegeinitiative ist der massive Fachkräftemangel, der sich aufgrund von Sparmassnahmen kombiniert mit der Einführung der neuen Bildungssystematik in den letzten zehn Jahren entwickelt hat. Namentlich bei den diplomierten Pflegefachpersonen ist der Mangel gross. Heute werden nur $43 \%$ des errechneten Bedarfs dieser Berufsleute ausgebildet, benötigt werden aber weit mehr. Daher kommen auf 3 Diplomabschlüsse zwei aus dem Ausland zugewanderte Personen. Der Politik ist es nicht gelungen, wirksame Massnahmen gegen den Mangel zu ergreifen. Der SBK hat deshalb im Januar 2017 die Volksinitiative für eine starke Pflege lanciert.

Die Pflegeinitiative zielt mit verschiedenen Forderungen darauf ab, im Interesse der Patienten und ihrer Sicherheit mehr junge Menschen für einen Beruf im Pflegebereich zu motivieren und die Berufsleute im Arbeitsprozess zu halten. Namentlich sollen die Anzahl Ausbildungsabschlüsse zur diplomierten Pflegefachperson mit finanziellen Anreizen erhöht werden, die Berufsrollen klar definiert und alle in der Pflege tätigen Personen ausbildungs- und kompetenzgerecht eingesetzt werden. Mit den direkt bei den Krankenkassen in Rechnung gestellten, eigenverantwortlich erbrachten Pflegeleistungen werden die beruflichen Kompetenzen der Pflegefachpersonen anerkannt. Zur Attraktivitätssteigerung gehören in diesem typischen Frauenberuf auch individuelle Arbeitszeitmodelle und mehr Plätze für die ausserfamiliäre Kinderbetreuung. Laufbahn- und Lohnperspektiven, die derVerantwortung und der geforderten und erbrachten Leistung entsprechen, tragen zum Personalerhalt bei.

bei der direkten Arbeit mit den Patienten in unterlassenen Gesprächen, unzureichenden Informationen und mangelnder Unterstützung in der Alltagsbewältigung zeigen werde [1]. Die zeitgleich erfolgte Bildungsreform (vgl. Kasten, OdASanté 2017) und die Umstrukturierung der Ausbildungsangebote liessen die Ausbildungsabschlüsse zusätzlich einbrechen. Der zukünftige Bedarf an Pflegepersonal konnte mit den ausgestellten Ausbildungsabschlüssen schon im Jahr 2008 nur zu rund 50\% gedeckt werden [2].

Sechs Jahre später hat sich die Situation nur unwesentlich verbessert. Während die Ausbildungsabschlüsse bei den Fachfrauen Gesundheit ${ }^{1}$ mit Eidgenössischem Fähigkeitszeugnis (FaGe EFZ) gemessen am errechneten Bedarf auf $84 \%$ erhöht werden konnten, sanken die Ausbildungsabschlüsse bei den diplomierten Pflegefachpersonen (früher diplomierte Krankenschwestern) im selben Zeitraum nochmals um rund $10 \%$ auf nunmehr 43\% [3]. Es ist die logische Folge, dass auf drei inländische Diplomabschlüsse im Zeitraum von 20102014 zwei aus dem Ausland zugewanderte Personen kamen [4].

\section{Politische Reaktion auf den Fachkräfte- mangel}

Aufgrund der Entwicklungen bei den Pflegeberufen forderten verschiedene Parlamentarier dringend dazu auf, mit konkreten Massnahmen den Berufen Anerkennung zu zollen und attraktivitätssteigernde Massnahmen zu ergreifen [5]. Mit einer parlamentarischen Initiative schlug Nationalrat Joder vor, die Arbeit der 


\section{OdASanté}

\section{SCHWEIZERISCHE BILDUNGSSYSTEMATIK}

Höhere Berufsbildung

Eidg. Berufsbildungsgesetz
Hochschulen

Hochschulförderungs- und -koordinationsgesetz

weitere Hochschulgesetze

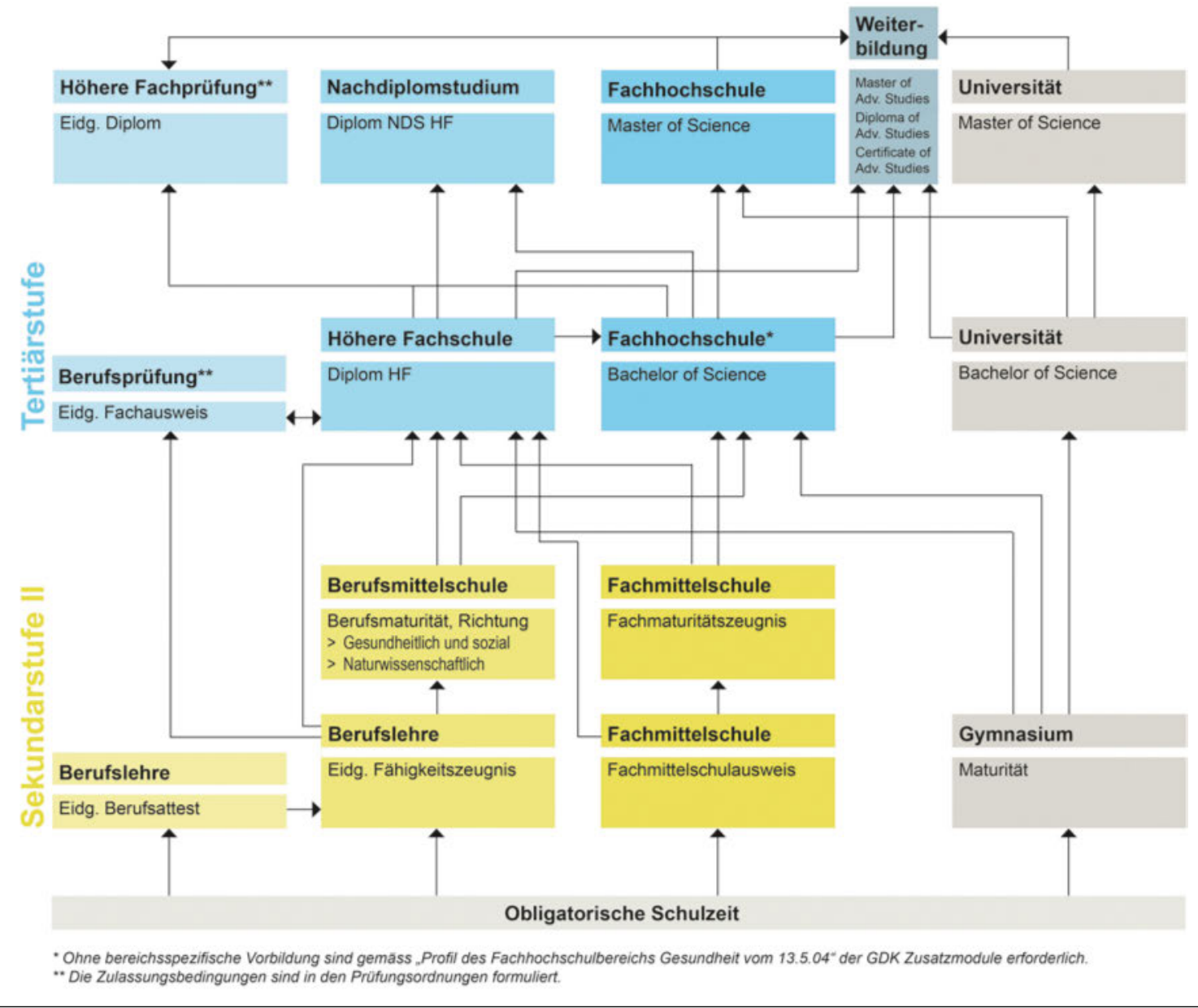

OdASanté, Schweizerische Bildungssystematik.

Pflegefachpersonen in einen eigenverantwortlichen und in einen delegierten Bereich zu unterscheiden. Im eigenverantwortlichen Bereich sollte die Pflegefachperson in Zukunft ihre Leistungen der Krankenkasse direkt in Rechnung stellen können, ohne Unterschrift einer Ärztin. Viele Politiker waren diesem Gesetzesvorschlag wohlgesinnt, trotzdem lehnte der Nationalrat das Eintreten auf das Geschäft im April 2016 ab, ohne darüber diskutiert zu haben.

\section{Weshalb eine Volksinitiative}

Mit dem Masterplan «Bildung Pflegeberufe» beabsichtigten Bund, Kantone und OdASanté, die Zahl der Berufsabschlüsse zu erhöhen. Bei den Berufsabschlüssen EFZ ist dies gelungen. Der offiziellen Verlautbarung, dass die Anzahl der Pflegefachkräfte dank dem Masterplan gesteigert werden konnte [6], kann aber nicht zugestimmt werden, im Gegenteil; diese sind nochmals 
gesunken. Konkret konnten in den letzten 5 Jahren 10000 Pflegefachdiplome zu wenig ausgestellt werden. Da diese Tatsache offensichtlich weder im Parlament noch beim Bundesrat weiter Beachtung fand und keine wirksamen Massnahmen zur Verbesserung der Situation ergriffen wurden, entschied sich der SBK, nach aussen zu treten, bevor der Schaden noch grösser wird. Denn die tiefen Ausbildungszahlen bei den diplomierten Pflegefachpersonen lassen sich auf die Dauer nicht mit ausländischen Kolleginnen kompensieren, und der Mangel kann die Sicherheit der Patienten gefährden. Dies belegen verschiedene wissenschaftliche Untersuchungen. Ein Ergebnis daraus: Wenn der Anteil der diplomierten Pflegefachpersonen um 10\% steigt, liegt die Mortalität um 11\% tiefer [7].

\section{Inhalt der Pflegeinitiative}

Das Ziel der Volksinitiative ist es, die pflegerische Versorgung im Interesse der Patienten und ihrer Sicherheit auch in Zukunft sicherzustellen.

Die Initiative fordert, dass in die Aus- und Weiterbildungen der Pflegepersonen investiert wird, wobei die Ausbildungszahlen bei den diplomierten Pflegefachpersonen mit entsprechenden Anreizen dringend erhöht werden müssen. Vonnöten sind weiter klar definierte Berufsrollen, ausbildungs- und kompetenzgerechter Einsatz aller in der Pflege tätigen Personen, Laufbahnmöglichkeiten sowie Lohnperspektiven, die der Verantwortung und der Leistung entsprechen.

Um die Pflegepersonen länger im Beruf zu halten, müssen auch die Rahmenbedingungen attraktiver werden. Dazu gehören in diesem typischen Frauenberuf familienfreundlichere Bedingungen mit individuellen Arbeitszeitmodellen und mehr Plätzen für die ausserfamiliäre Kinderbetreuung.

Last but not least sollen die Pflegefachpersonen typische Pflegeleistungen wie beispielsweise die Feststellung des Pflegebedarfs, die Koordinations- und Beratungstätigkeiten sowie die grundpflegerischen Tätigkeiten direkt mit den Krankenkassen abrechnen können. Dies wertet den Beruf auf und macht ihn attraktiver. Für die fach- und sachgerechte Ausführung dieser Tätigkeiten können die Pflegefachpersonen bereits heute rechtlich zur Verantwortung gezogen werden. Es handelt sich somit um aktuell geltende, berufspraktische Kompetenzen der Pflegefachpersonen.

Die Unterschriftensammlung für die Initiative zeigt, dass sich die Bevölkerung um die pflegerische Versorgung in der Schweiz sorgt. Der SBK ist zuversichtlich, dass die Initiative in den nächsten Monaten eingereicht werden kann.

\section{Literatur}

1 Schubert M, Schaffert-Vitfliet B, De Geest S, Aiken L, Sloane DM, Clark S, Abraham I \& Glass T (2005). RICH - Nursing Study. Rationing of Nursing Care in Switzerland $=\mathrm{CH}$. Effects of Rationing of Nursing Care in Switzerland on Patients and Nurses Outcomes. Schlussbericht Bundesamt für Gesundheit.

2 Schweizerische Konferenz der Gesundheitsdirektorinnen und Gesundheitsdirektoren GDK / Organisation der Arbeitswelt Gesundheit OdASanté (2009). Nationaler Versorgungsbericht für die Gesundheitsberufe. Personalbedarf und Massnahmen zur Personalsicherung auf nationaler Ebene.

3 Schweizerische Konferenz der Gesundheitsdirektorinnen und Gesundheitsdirektoren GDK/Organisation der Arbeitswelt Gesundheit OdASanté (2016). Nationaler Versorgungsbericht für die Gesundheitsberufe. Personalbedarf und Massnahmen zur Personalsicherung auf nationaler Ebene.

4 Merçay C, Burla L, Widmer M (2016). Gesundheitspersonal in der Schweiz. Bestandesaufnahme und Prognosen bis 2030. Obsan Bericht 71. Neuchâtel, Schweizerisches Gesundheitsobservatorium.

5 Heim, Bea. Postulate 10.3127 und 10.3128 vom 16.3.2010 «Sicherung der Alterspflege» und "Attraktivität der Krankenpflege-Ausbildung»; Aubert, Josiane. Interpellation 10.3513 vom 17.6.2010 «Ausbildung von Pflegepersonal auf den Tertiärstufen A und B. Transparenz bei den Eintritten"; Joder, Rudolf. Parlamentarische Initiative 11.418 vom 16.3.2011 «Gesetzliche Anerkennung der Verantwortung der Pflege»; CVP-Fraktion, Motion 11.3889 vom 29.9.2011 «Umschulungsmöglichkeiten und Zweitausbildungen für Pflegepersonal fördern und unterstützen».

6 Staatssekretariat für Bildung, Forschung und Innovation SBFI, Medienmitteilung vom 3.2.2016, https://www.sbfi.admin.ch/sbfi/ de/home/aktuell/medienmitteilungen/news-anzeige-nsb.msgid-60542.html.

7 Aiken LH, Sloan D, Griffiths P, Rafferty AM, Bruyneel L, McHugh M, Maier CB, Moreno-Casbas T, Ball JE, Ausserhofer D, Sermeus W, in: BMJ Quality \& Safty, Nursing skill mix in European hospitals: cross-sectional study of the association with mortality, patient ratings, and quality of care, November 15, 2016, http://dx.doi. org/10.1136/bmjqs-2016-005567. 